\title{
BMJ Open Multicentre prospective observational study of sodium-glucose cotransporter-2 inhibitor-associated postoperative ketoacidosis: the SAPKA study protocol
}

\author{
Hiroyuki Seki (D) , ${ }^{1}$ Norifumi Kuratani, ${ }^{2}$ Toshiya Shiga, ${ }^{3}$ Yudai Iwasaki, ${ }^{4}$ \\ Kanae Karita, ${ }^{5}$ Kazuki Yasuda, ${ }^{6}$ Tomoko Yorozu ${ }^{1}$
}

To cite: Seki H, Kuratani N, Shiga T, et al. Multicentre prospective observational study of sodium-glucose cotransporter-2 inhibitorassociated postoperative ketoacidosis: the SAPKA study protocol. BMJ Open 2021;11:e049592. doi:10.1136/ bmjopen-2021-049592

- Prepublication history and additional supplemental material for this paper are available online. To view these files, please visit the journal online (http://dx.doi.org/10.1136/ bmjopen-2021-049592)

Received 01 February 2021 Accepted 15 0ctober 2021

Check for updates

(C) Author(s) (or their employer(s)) 2021. Re-use permitted under CC BY-NC. No commercial re-use. See rights and permissions. Published by BMJ.

${ }^{1}$ Anesthesiology, Kyorin University, Mitaka, Japan ${ }^{2}$ Anesthesiology, Saitama Children's Medical Center,

Saitama, Japan

${ }^{3}$ Anesthesiology, International University of Health and Welfare Ichikawa Hospital, Ichikawa,

Japan

${ }^{4}$ Anesthesiology and

Perioperative Medicine, Tohoku University, Sendai, Japan

${ }^{5}$ Hygiene and Public Health, Kyorin University, Mitaka, Japan ${ }^{6}$ Diabetes, Endocrinology and Metabolism, Kyorin University, Mitaka, Japan

Correspondence to

Hiroyuki Seki;

hseki@ks.kyorin-u.ac.jp

\section{ABSTRACT}

Introduction Sodium-glucose cotransporter 2 (SGLT2) inhibitors are a new class of antihyperglycaemic agents that promote urinary glucose excretion in the renal proximal tubule and have cardio-protective and renalprotective properties. However, there are several safety concerns related to increased risks of hypoglycaemic, urinary tract infections and ketoacidosis. Ketoacidosis is a potentially fatal complication that often presents as euglycaemic ketoacidosis during SGLT2 inhibitor treatment. Furthermore, invasive treatment and related surgical stress may increase the risk of ketogenesis. Therefore, this study aims to clarify the incidence of SGLT2 inhibitor-associated postoperative ketoacidosis (SAPKA) among patients who are receiving SGLT2 inhibitors and undergoing surgery under general anaesthesia.

Methods and analysis This multicentre, prospective, observational study will recruit 750 adult Japanese patients with diabetes who are receiving SGLT2 inhibitors and undergoing surgery under general anaesthesia. Urine samples will be collected on postoperative days $0,1,2$ and 3. Blood gas analysis will be performed when urine ketone positivity is detected. The incidence of postoperative ketoacidosis will be identified based on urine ketone positivity and a blood $\mathrm{pH}$ of $\leq 7.3$. The study will also collect data to identify risk factors for SAPKA.

Ethics and dissemination The study protocol has been approved by the ethics committee of Kyorin University (approval number: 785, 26 0ctober 2020) and local ethical approval will be required at each participating centre. Study findings will be submitted to peer-reviewed journals and abstracts will be submitted to relevant national and international meetings.

Trial registration number UMIN000042795

\section{INTRODUCTION}

Sodium-glucose cotransporter 2 (SGLT2) inhibitors are a new class of antihyperglycaemic agents for treating diabetes. These agents promote urinary glucose excretion via inhibition of SGLT2 that is expressed in the kidney's proximal tubule. 'Unlike conventional antidiabetic agents, such as sulfonylureas, SGLT2 inhibitors exert their
Strengths and limitations of this study

- This is the first prospective study to explore the incidence of sodium-glucose cotransporter 2 (SGLT2) inhibitor-associated postoperative ketoacidosis among patients undergoing surgery under general anaesthesia.

- More accurately identifies ketoacidosis, relative to previous studies, by actively monitoring for its development.

- Unable to confirm a causal relationship between SGLT2 inhibitors and postoperative ketoacidosis.

- May underestimate the incidence of ketoacidosis, which is identified based on urine ketone positivity.

glucoregulatory effects independent of insulin secretion, which reduces the risk of developing hypoglycaemic. Since the first approval in the USA during 2013, four classes of SGLT2 inhibitors (canagliflozin, dapagliflozin, empagliflozin and ertugliflozin) have been approved by the US Food and Drug Administration (FDA) and three other classes (ipragliflozin, luseogliflozin and tofogliflozin) have been approved in Japan. Recent large-scale clinical trials have demonstrated that SGLT2 inhibitors reduced the risks of myocardial infarction, heart failure, renal failure, cardiovascular mortality and all-cause mortality among patients with type 2 diabetes who had high cardiovascular risk. ${ }^{2-6}$ Thus, the use of SGLT2 inhibitors has been steadily increasing during recent years because of their effective glycaemic control and other benefits. In May 2020, the FDA approved dapagliflozin for treating heart failure with a reduced ejection fraction, regardless of the presence or absence of type 2 diabetes, which may further increase the prescription of SGLT2 inhibitors.

There are several safety concerns related to the use of SGLT2 inhibitors, including 
ketoacidosis as a potentially fatal complication. ${ }^{7}$ In 2015 , the FDA issued safety warnings regarding the risk of ketoacidosis that is associated with SGLT2 inhibitors. ${ }^{89}$ The mechanism underlying SGLT2 inhibitor-associated ketoacidosis is not entirely clear, although it may be related to insulin deficiency, carbohydrate deprivation, increased concentrations of counter-regulating hormones, hypovolemia and fasting. Furthermore, invasive treatment and related surgical stress may increase the risk of ketogenesis, which can develop without marked elevation of blood glucose levels. Thus, patients who receive SGLT2 inhibitors should be considered to have a risk of ketoacidosis during the perioperative period. ${ }^{10}$ However, SGLT2 inhibitor-associated postoperative ketoacidosis (SAPKA) may be underestimated because (i) it can present without significant hyperglycaemic ('euglycaemic ketoacidosis'); (ii) the predominant symptoms are nausea, vomiting, tachypnea and abdominal pain, which are common events in the postoperative setting and (iii) the diagnosis is based on blood gas analysis and measurements of ketone bodies, which are not routinely performed in clinical practice. ${ }^{11}$ Therefore, given the increasing use of SGLT2 inhibitors, there is an urgent need to evaluate the 'real-world' safety of SGLT2 inhibitors. The SAPKA study aims to explore the incidence of SAPKA among surgical patients who are receiving SGLT2 inhibitors, and to identify its risk factors.

\section{METHODS AND ANALYSIS \\ Study objectives}

This study's primary aim is to explore the incidence of SAPKA among patients with diabetes who are receiving SGLT2 inhibitors and undergoing surgery under general anaesthesia. The secondary aim is to identify potential risk factors for the development of SAPKA in this setting.

\section{Study design and setting}

This prospective, multicentre, observational study will be performed at 17 Japanese university hospitals: Akita University Hospital (Akita), Fukui University Hospital (Fukui), Fukuoka University Hospital (Fukuoka), Hamamatsu University Hospital (Shizuoka), Hirosaki University Hospital (Aomori), the Jikei University Hospital (Tokyo), Kawasaki Medical School Hospital (Okayama), Kumamoto University Hospital (Kumamoto), Kyorin University Hospital (Tokyo), Kyushu University Hospital (Fukuoka), University of Miyazaki Hospital (Miyazaki), Nihon University Hospital (Tokyo), Sapporo Medical University Hospital (Hokkaido), Shinshu University Hospital (Nagano), Tohoku University Hospital (Miyagi), Tokyo Dental College Ichikawa General Hospital (Chiba) and Yamagata University Hospital (Yamagata). The study protocol is registered in the University Hospital Medical Information Network Clinical Trials Registry, which is a non-profit organisation in Japan, and complies with the requirements of the International Committee of Medical

\section{Box 1 Eligibility criteria}

Inclusion criteria
1. Written informed consent.
2. Age of $\geq 20$ years.
3. Diagnosis of diabetes.
4. Planned surgery under general anaesthesia (elective or emergent).
5. Receiving one of the following SGLT2 inhibitors:
Apleway tablets (tofogliflozin).
Canaglu tablets (canagliflozin).
Canalia combination tablets (canagliflozin/teneligliptin).
Deberza tablets (tofogliflozin).
Forxiga tablets (dapagliflozin).
Jardiance tablets (empagliflozin).
Lusefi tablets (luseogliflozin).
Suglat tablets (ipragliflozin).
Sujanu combination tablets (ipragliflozin/sitagliptin).
Tradiance combination tablets (empagliflozin/linagliptin).
Exclusion criteria
1. Diagnosed with ketoacidosis at the screening or anaesthesia
induction.
2. Stopped receiving SGLT2 inhibitors>1 week before surgery.
3. Surgery was performed without general anaesthesia.
SGLT2, sodium-glucose cotransporter 2 .

Journal Editors. Study recruitment commenced on 4 January 2021.

\section{Eligibility criteria}

Subjects will be enrolled if they fulfil all the inclusion criteria and none of the exclusion criteria, which are described in Box 1.

\section{Patient and public involvement}

There was no patient or public involvement in the design and conduct of this study.

\section{Outcome measures}

The primary outcome measure is the incidence of postoperative ketoacidosis during the first 3 days after surgery. Ketoacidosis is defined as urine ketone positivity and a blood $\mathrm{pH}$ of $<7.3$. The secondary outcomes are the risk factors for the development of postoperative ketoacidosis.

\section{Data collection and data management}

Baseline data will be collected by a trained investigator after the recruitment session. Figure 1 shows a summary of the study protocol. Urine samples will be collected on postoperative days (PODs) 0, 1, 2 and 3. If urine ketone positivity is detected, arterial or venous blood will be collected to measure blood $\mathrm{pH}$, bicarbonate concentration, glucose concentration and electrolytes, including sodium, potassium and chloride. After POD3, the investigator at each site will determine whether the patient developed ketoacidosis by checking the patient's medical records until discharge. Data will be collected using a paper-based case report form (CRF) that was designed specifically for this study (online supplemental figures la,b). Patient privacy will be protected by assigning each 


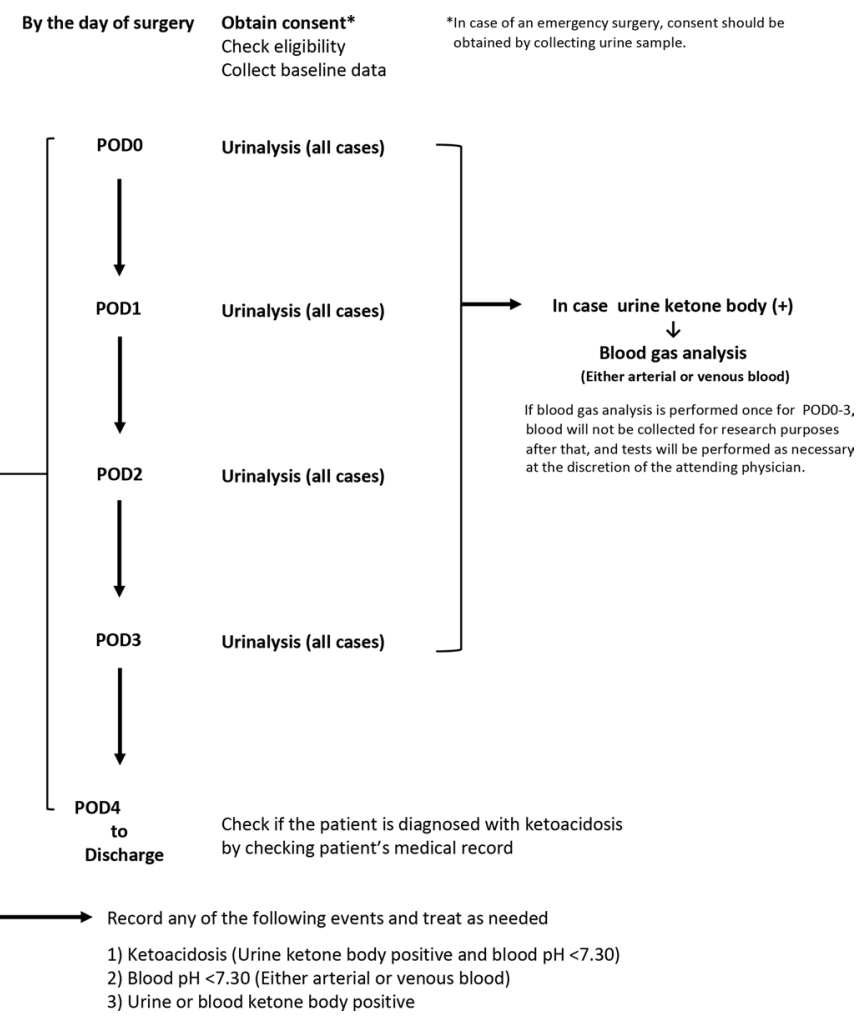

Figure 1 Summary of protocol. POD, postoperative day.

participant a unique study identification number at the time of recruitment. The study identification numbers will be kept separate from the study data and will only be accessible to members of the SAPKA study team.

After patient discharge, the investigator will complete the CRF. A data manager at each site will audit the data quality and enter the data in the UMIN Internet Data and Information system for Clinical and Epidemiological research (UMIN-INDICE). The UMIN-INDICE is a secure web-based registry system provided by the UMIN that aims to store anonymised case data from clinical research conducted by individual researchers. The data are available to third parties and may be used by qualified researchers to inspect the case data or to repeat statistical analyses. All anonymised data will be subsequently analysed by a statistical analysis team, which is independent from the data collection team.

\section{Data sharing statement}

Anonymised patient data will be made available via the UMIN-INDICE to qualified investigators who submit an approved research proposal.

\section{Statistical analysis}

No formal power calculation was performed because this is the first prospective study of SAPKA and we are not aware of any previous epidemiological reports regarding SAPKA. According to previous studies of insurance claims and adverse event data, ${ }^{12-16}$ we hypothesise that the incidence of SAPKA will be $>0.5 \%$ among patients who regularly receive SGLT2 before surgery. Using the conventional rule of three for a Poisson distribution, ${ }^{17}$ we assume that $\geq 600$ patients will be needed to find $\geq 1$ patient who experiences SAPKA with $95 \%$ confidence under the $0.5 \%$ incidence assumption. We also estimated a missing data rate of approximately $20 \%$ and ultimately aimed to recruit approximately 750 patients.

The patients' baseline characteristics and perioperative variables will be summarised using standard descriptive statistics. The incidence of SAPKA will be calculated as the number of patients fulfilling the SAPKA diagnostic criteria divided by the total number of participants with data from at least one postoperative urinalysis. The result (\%) will be reported with the $95 \%$ CI. Based on the anticipated small number of SAPKA cases, sensitivity analysis will be performed using bootstrap resampling methods.

If $>10 \%$ of recruited patients do not have postoperative urinalysis data, we will impute missing data using the multiple imputation by chained equations method. ${ }^{18}$ The number of imputations will be determined based on the percentages of missing values, and the results for the imputations will be pooled using Rubin's rule.

If we identify $>10$ SAPKA cases during the study period, we will conduct a nested case-control analysis to explore the perioperative variables that are associated with SAPKA. Multivariable logistic regression models will be constructed to identify any risk factors for SAPKA, and perioperative variables with high clinical relevance will be incorporated into the multivariable models. Statistical significance will be identified based on a two-sided p-value of $<0.05$. Statistical analysis will be performed using R, SPSS, and SPBS software.

\section{ETHICS AND DISSEMINATION}

\section{Ethical approval}

The study will be conducted in accordance with the Declaration of Helsinki. The study protocol has been approved by the ethics committee of Kyorin University (approval number: 785, 26 October 2020) and local ethical approval will be required at each participating centre. Informed consent will be requested from all patients, although the study will not affect their clinical management.

\section{Dissemination}

The findings of this study will be submitted to peerreviewed journals and abstracts will be submitted to relevant national and international meetings. If interim analysis reveals a lower limit of $95 \%$ CI with an incidence of $>1 \%$ for postoperative ketoacidosis, that result will also be submitted to peer-reviewed journals.

\section{DISCUSSION}

Diabetic ketoacidosis (DKA) is an acute diabetic complication that is rare but potentially life-threatening. This complication is characterised by hyperglycaemic, increased blood ketone concentrations and metabolic acidosis, which are related to absolute or relative insulin deficiency and an increase in counter-regulatory 
hormones. ${ }^{19}$ Surgery and prolonged fasting may precipitate the development of SGLT2 inhibitor-associated ketoacidosis, ${ }^{15}$ and a systematic review of perioperative SGLT2 inhibitor-associated ketoacidosis revealed that $89 \%$ of patients presented with euglycaemic ketoacidosis (blood glucose concentration of $<250 \mathrm{mg} / \mathrm{dL}) .{ }^{11}$ The most common symptoms at presentation are nausea, vomiting, tachypnea and abdominal pain, although postoperative patients without ketoacidosis also commonly experience these symptoms. Therefore, it can be challenging to diagnose SGLT2 inhibitor-associated ketoacidosis in surgical patients. Several guidelines also have variable diagnostic criteria for DKA, although the diagnosis should generally be based on data regarding blood $\mathrm{pH}$, urine or blood ketone positivity and blood glucose concentration. ${ }^{20}$ However, blood glucose concentration is not helpful for identifying SGLT2 inhibitor-associated ketoacidosis. Thus, we defined ketoacidosis as urine ketone positivity and a blood $\mathrm{pH}$ of $<7.3$.

The SAPKA study has several strengths. First, this study prospectively explores the incidence of SAPKA among patients undergoing surgery under general anaesthesia, which may help address underestimation of its incidence using adverse event reports. ${ }^{12-16}$ Thus, our reported incidence may be more accurate because we will actively determine whether all participants develop ketoacidosis. Second, the multicentre study design will help increase the generalisability of the results. Third, we will attempt to identify the risk factors for developing SAPKA, which may help improve the perioperative management of patients who are receiving SGLT2 inhibitors.

The study also has several potential limitations. First, the study cannot confirm a causal relationship between SGLT2 inhibitors and postoperative ketoacidosis, as perioperative DKA may also be caused by various precipitating factors, including the surgery itself, prolonged fasting and other antidiabetic agents. However, this study aims to estimate the crude incidence of SAPKA in a prospective manner, rather than confirming a causal relationship. Second, measuring urine ketone concentrations may underestimate the extent of ketoacidosis, as the most commonly used urine assay only measures one type of ketone bodies (acetoacetate). ${ }^{21}$ The American Association of Clinical Endocrinologists and the American College of Endocrinology recommend measuring blood $\beta$-hydroxybutyrate concentrations to diagnose DKA. ${ }^{22}$ Although ketonuria does not necessarily imply ketoacidosis, urine ketone measurement is more practical because blood ketone measurements require several days in most Japanese hospitals.

The results from the SAPKA study will provide new evidence regarding safety concerns associated with perioperative use of SGLT2 inhibitors, which may help guide the development of strategies for managing surgical patients who are receiving SGLT2 inhibitors.

Contributors HS conceived the study. All authors designed the study. HS drafted the protocol. NK, TS, YI, KK, KY and TY revised the protocol. HS and NK drafted the manuscript. TS, YI, KK, KY and TY revised the manuscript critically. All authors read and approved the final manuscript.

Funding This work is supported by the Japan Society for the Promotion of Science Grant-in-Aid for Scientific Research (C) (Grant Number 21K06676).

Competing interests Dr Yasuda reports grants from Ono Pharmaceutical.

Patient consent for publication Not required.

Provenance and peer review Not commissioned; externally peer reviewed.

Supplemental material This content has been supplied by the author(s). It has not been vetted by BMJ Publishing Group Limited (BMJ) and may not have been peer-reviewed. Any opinions or recommendations discussed are solely those of the author(s) and are not endorsed by BMJ. BMJ disclaims all liability and responsibility arising from any reliance placed on the content. Where the content includes any translated material, BMJ does not warrant the accuracy and reliability of the translations (including but not limited to local regulations, clinical guidelines, terminology, drug names and drug dosages), and is not responsible for any error and/or omissions arising from translation and adaptation or otherwise.

Open access This is an open access article distributed in accordance with the Creative Commons Attribution Non Commercial (CC BY-NC 4.0) license, which permits others to distribute, remix, adapt, build upon this work non-commercially, and license their derivative works on different terms, provided the original work is properly cited, appropriate credit is given, any changes made indicated, and the use is non-commercial. See: http://creativecommons.org/licenses/by-nc/4.0/.

ORCID iD

Hiroyuki Seki http://orcid.org/0000-0001-5311-5088

\section{REFERENCES}

1 Gallo LA, Wright EM, Vallon V. Probing SGLT2 as a therapeutic target for diabetes: basic physiology and consequences. Diab Vasc Dis Res 2015;12:78-89.

2 Zinman B, Wanner C, Lachin JM, et al. Empagliflozin, cardiovascular outcomes, and mortality in type 2 diabetes. N Engl J Med 2015;373:2117-28.

3 Wanner C, Inzucchi SE, Lachin JM, et al. Empagliflozin and progression of kidney disease in type 2 diabetes. $N$ Engl $J$ Med 2016;375:323-34.

4 Neal B, Perkovic V, Mahaffey KW, et al. Canagliflozin and cardiovascular and renal events in type 2 diabetes. N Engl J Med 2017;377:644-57.

5 McMurray JJV, Solomon SD, Inzucchi SE, et al. Dapagliflozin in patients with heart failure and reduced ejection fraction. $N$ Engl $J$ Med 2019;381:1995-2008.

6 Perkovic V, Jardine MJ, Neal B, et al. Canagliflozin and renal outcomes in type 2 diabetes and nephropathy. N Engl $\mathrm{J} \mathrm{Med}$ 2019;380:2295-306.

7 Peters AL, Buschur EO, Buse JB, et al. Euglycemic diabetic ketoacidosis: a potential complication of treatment with sodiumglucose cotransporter 2 inhibition. Diabetes Care 2015;38:1687-93.

8 U.S. Food and Drug Administration. FDA drug safety communication: FDA warns that SGLT2 inhibitors for diabetes may result in a serious condition of too much acid in the blood, 2015. Available: https:// wayback.archive-it.org/7993/20170112031553/http://www.fda.gov/ Drugs/DrugSafety/ucm446845.htm [Accessed 05 Jan 2021].

9 U.S. Food and Drug Administration. FDA drug safety communication: FDA revises labels of SGLT2 inhibitors for diabetes to include warnings about too much acid in the blood and serious urinary tract infections, 2015. Available: https://www.fda.gov/drugs/drugsafety-and-availability/fda-revises-labels-sglt2-inhibitors-diabetesinclude-warnings-about-too-much-acid-blood-and-serious\#: :text= Additional\%20Information\%20for\%20Patients,and\%20serious\% 20urinary\%20tract\%20infections [Accessed 05 Jan 2021].

10 Preiser J-C, Provenzano B, Mongkolpun W, et al. Perioperative management of oral glucose-lowering drugs in the patient with type 2 diabetes. Anesthesiology 2020;133:430-8.

11 Thiruvenkatarajan V, Meyer EJ, Nanjappa N, et al. Perioperative diabetic ketoacidosis associated with sodium-glucose cotransporter-2 inhibitors: a systematic review. Br J Anaesth 2019;123:27-36.

12 Fralick M, Schneeweiss S, Patorno E. Risk of diabetic ketoacidosis after initiation of an SGLT2 inhibitor. N Engl J Med 2017;376:2300-2.

13 Blau JE, Tella SH, Taylor SI, et al. Ketoacidosis associated with SGLT2 inhibitor treatment: analysis of FAERS data. Diabetes Metab Res Rev 2017;33:10.1002/dmrr.2924. 
14 Ueda P, Svanström H, Melbye M, et al. Sodium glucose cotransporter 2 inhibitors and risk of serious adverse events: nationwide register based cohort study. BMJ 2018;363:k4365.

15 Meyer EJ, Gabb G, Jesudason D. SGLT2 inhibitor-associated euglycemic diabetic ketoacidosis: a South Australian clinical case series and Australian spontaneous adverse event notifications. Diabetes Care 2018;41:e47-9.

16 Douros A, Lix LM, Fralick M, et al. Sodium-Glucose Cotransporter-2 inhibitors and the risk for diabetic ketoacidosis : a multicenter cohort study. Ann Intern Med 2020;173:417-25.

17 Onakpoya IJ. Rare adverse events in clinical trials: understanding the rule of three. BMJ Evid Based Med 2018;23:6.

18 Buuren S, Groothuis-Oudshoorn K. Mice : Multivariate Imputation by Chained Equations in R. J Stat Softw 2011;45:1-67.
19 Kitabchi AE, Umpierrez GE, Miles JM, et al. Hyperglycemic crises in adult patients with diabetes. Diabetes Care 2009;32:1335-43.

20 Lee K, Park IB, Yu SH, et al. Characterization of variable presentations of diabetic ketoacidosis based on blood ketone levels and major Society diagnostic criteria: a new view point on the assessment of diabetic ketoacidosis. Diabetes Metab Syndr Obes 2019;12:1161-71.

21 Sacks DB, Arnold M, Bakris GL, et al. Guidelines and recommendations for laboratory analysis in the diagnosis and management of diabetes mellitus. Diabetes Care 2011;34:e61-99.

22 Handelsman Y, Henry RR, Bloomgarden ZT, et al. American association of clinical endocrinologists and American College of endocrinology position statement on the association of SGLT-2 inhibitors and diabetic ketoacidosis. Endocr Pract 2016;22:753-62. 